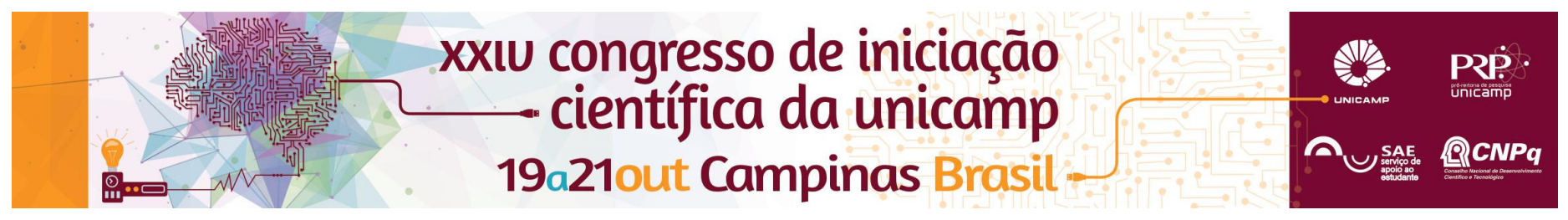

\title{
Characterization of Stearoyl-CoA Desaturase 2 (SCD2) in the brain of Rodents
}

Felipe C. Silva*, Roberta H. Tóvolli, Joseane Morari, Lucas F. R. Nascimento, Daniela S. Razolli, Licio A. Velloso.

\section{Abstract}

Steraroyl-CoA Desaturase (SCD) is a key enzyme in the lipogenesis de novo. SCD2 is the main isoform in the murine central nervous system (CNS), however its anatomical or cellular distribuition is currently unknow. In this work we evaluated SCD2 distribution in ten anatomical regions (spinal cord, cerebellum, cortex, hippocampus, olfactory bulb, thalamus, hypothalamus, midbrain and striatum) of CNS. SCD2 is more abundant in spinal cord, midbrain and hypothalamus and is also present in all cell types analysed (microglia, astrocytes and neurons), including those involved in proliferation (Ki67 and FGF10 positive cells). Thus SCD2 is highly expressed in the murine CNS, and it is expressed in all cell types. All experimental procedures involving mice were approved by the Ethics Committee at the University of Campinas (4138-1) and supported by FAPESP.

\section{Key words:}

SCD2, Hypothalamus, Proliferation

\section{Introduction}

De novo lipogenesis (DNL) is a metabolic pathway involved in the conversion of lipids into carbohydrates. This process is highly regulated according to the cell needs. Stearoyl-CoA Desaturase (SCD) is a key enzyme in DNL, once it regulates monoiunsaturated fatty acids (MUFAs) production which are essencial for many cellular functions, including cell membrane formation.

In rodents there are four isoforms of SCD and SCD2 is the main $\delta 9$ desaturase in the murine central nervous system (CNS), although its cellular and anatomical distribuition is currently unknow.

In this work, we aimed to characterize SCD2 distribution in the mice brain by gene expression immunofluorescence analysis.

\section{Results and Discussion}

SCD2 was expressed in at least ten different anatomical regions of CNS (spinal cord, cerebellum, cortex, hippocampus, olfactory bulb, thalamus, hypothalamus, midbrain and striatum). The highest expression levels of SCD2 were observed in spinal cord, midbrain and hypothalamus (Figure 1).

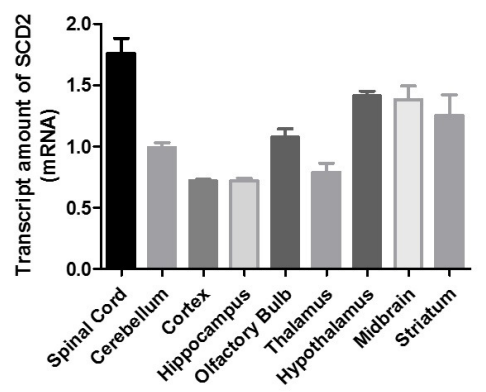

Figure 1: SCD2 gene expression in different regions of CNS.

SCD2 was also present in all cell types analysed: neurons, microglia and astrocytes. In hypothalamus, a novel neurogenic niche (Sousa-Ferreira et al., 2014), SCD2 is also present in proliferative cells expressing Ki67/FGF10+ (Figure 2).

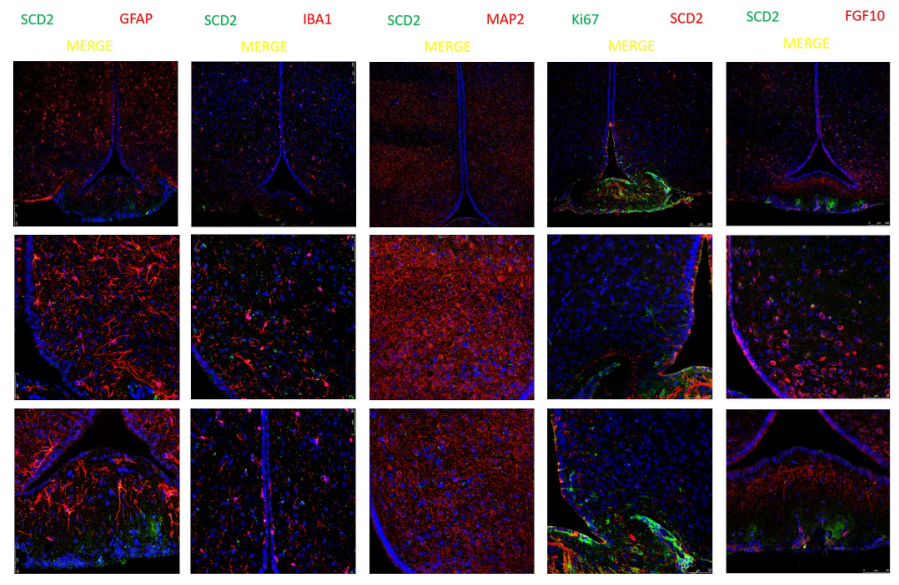

Figure 2: Hypothalamic distribution of SCD2 by immunofluorescence. Confocal images acquired using LEICA TCS SP5 II.

Thus SCD2 is broadly expressed in the murine CNS. Is present in all cell types analysed, with highlits for astrocyes. In the mediobasal hypothalamus, SCD2 is present in proliferative cells as well, indicating a possible role for SCD2 in stem cell maintanence/function.

\section{Conclusions}

SCD2 is expressed in at least ten anatomical structures of SNC, specially the hypothalmus, and in all cell types including those involved in cellular proliferation, suggesting its potential role in hypothalamic neurogenesis.

\section{Acknowledgement}

All experiments were supported by FAPESP/OCRC and CNPq

L. Sousa-Ferreira, L.P. de Almeida, C. Cavadas. Role of hypothalamic neurogenesis in feeding regulation. Trends Endocrinol. Metab., 25 (2014), pp. 80-88

Spector, A. A., Yorek, M. A. (1985). Lipid Membrane composition and cellular function.J. Lipid Res. Sep;26(9):1015-35. 